\title{
Tagraxofusp for the Treatment of Blastic Plasmacytoid Dendritic Cell Neoplasm (BPDCN): A Brief Report on Emerging Data
}

This article was published in the following Dove Press journal: OncoTargets and Therapy

\author{
Guillaume Beziat (iD) \\ Loïc Ysebaert iD ${ }^{1,2}$ \\ 'Hematology Department, University \\ Hospitals of Toulouse, IUC Toulouse- \\ Oncopole, Toulouse, France; ${ }^{2}$ University \\ Toulouse-3 Paul Sabatier, Toulouse, \\ France
}

\begin{abstract}
Blastic plasmacytoid dendritic cell neoplasm (BPDCN) is a rare myeloid malignancy, for which conventional chemotherapy has poor outcomes. CD123, the $\alpha$-subunit of interleukin (IL)-3 receptor, is constantly overexpressed at the surface of tumoral cells. Tagraxofusp (or SL-401) is a recombinant cytotoxin which consists of human interleukin-3 fused to a truncated diphtheria toxin. It is currently the only novel therapy with a prospective evaluation of efficacy and safety in the treatment of BPDCN and is also the only one to achieve FDA approval. In this short review, the results of tagraxofusp are summarized and perspectives of its use in BPDCN and in other malignancies are discussed. The safety profile is also summarized, since capillary leak syndrome is the main toxic effect of the drug, along with more common toxicities including an increase in transaminases and thrombocytopenia
\end{abstract} Keywords: CD123, blastic plasmacytoid dendritic cell neoplasm, targeted therapy, SL-401

\section{23: The $\alpha$-Subunit of IL-3 Receptor Is an Emerging Target in Hematologic Malignancies}

CD123 is the $\alpha$-subunit of interleukin (IL)-3 receptor. This receptor belongs to the Common Beta $\left(\beta_{\mathrm{C}}\right)$ cytokine receptor subfamily with IL-5 and GranulocyteMacrophage Colony Stimulating Factor receptors. All these membrane receptors are expressed at the surface of myeloid progenitors and play a key role in the regulation of hematopoiesis and inflammatory response. CD123 is expressed on the majority of CD34+ hematopoietic progenitors, but its expression is sustained only in the monocytic and granulocytic lineages. CD123 can be found at the surface of non-classical monocytes, but not granulocytes. It is involved in the proliferation and differentiation of myeloid progenitors, notably through the activation of the JAK/ STAT pathway after the reception of IL-3. The expression of CD123 is also found in hematologic malignancies, especially myeloid neoplasms such as acute myeloid leukemia (AML), hairy cell leukemia and blastic plasmacytoid dendritic cell neoplasm (BPDCN). ${ }^{1}$ Its expression on islands of clonally related plasmacytoid dendritic cells (pDC) in the bone marrow of $20 \%$ of chronic myelomonocytic leukemia patients is correlated to an increased risk of AML transformation. ${ }^{2} \mathrm{CD} 123$ was first reported to be a potential target in hematologic disease by Jordan and colleagues in 2000. They demonstrated that the interleukin-3 receptor alpha chain is expressed in CD34+/CD38- leukemic stem cells in AML, whereas it is absent at the surface of normal bone marrow-derived CD34+/CD38- cells. ${ }^{3}$ Moreover, a high expression
Correspondence: Guillaume Beziat Service d'Hématologie, Institut Universitaire du Cancer de Toulouse Oncopole, I Avenue Irène Joliot-Curie, Toulouse Cedex 9 31059, France

Tel +33531156355

Fax +3356177754

Email guillaume.beziat@hotmail.fr 
level of CD123 has been shown to be involved in enhanced proliferation and poorer outcomes in several hematologic neoplasms. ${ }^{4}$ Therefore, CD123 is one of the major therapeutic targets in the current development of new therapies in myeloid malignancies. 5,6

\section{Tagraxofusp: Poisoning Tumoral Cells Through CDI 23 Expression}

Tagraxofusp (or SL-401) is a recombinant protein which consists of the diphtheria toxin (DT) without its C-terminal binding domain fused to interleukin-3 (IL-3). This last part of the protein binds to CD123 at the surface of tumor cells. As a result, diphtheria toxin is internalized inside the CD123 expressing tumor cells. It impairs protein synthesis and triggers cell death, notably by Elongation Factor 2 (EF2) inhibition. ${ }^{7}$

Tagraxofusp is currently the only novel therapy to receive FDA approval for the treatment of BPDCN in adult and pediatric patients 2 years and older. ${ }^{8}$ The product received EMA orphan medicinal product designation in February 2019. Targeting CD123 in the treatment of BPDCN is particularly relevant since BPDCN is characterized by a constant overexpression of CD123 on the surface of tumor cells. ${ }^{9}$ It provided the rationale to investigate the efficacy of tagraxofusp in BPDCN.

\section{BPDCN: A Rare and Highly Refractory Hematologic Malignancy} Clinical Characteristics and Diagnosis

According to the World Health Organization (WHO) 2008 guidelines, BPDCN was classified in the acute myeloid leukemia (AML)/related family of neoplasms. However, following the discovery that it may originate from type 2 dendritic cells (plasmacytoid), classification under WHO 2016 guidelines placed BPDCN in its own category among myeloid malignancies. ${ }^{10}$

BPDCN is a rare and aggressive myeloid malignancy with poor outcomes. It mainly affects patients over 60 years of age. Skin is the organ most frequently involved, but BPDCN can also spread to bone marrow, lymph nodes and the central nervous system (CNS). Rapid and aggressive progression to the terminal leukemic phase is common. ${ }^{11}$

The diagnosis is based on the identification of clonal cells derived from precursors of plasmacytoid dendritic cells in a sample of an involved organ. Tumor cells must express at least four of five pDCs-specific markers (CD4, CD56, CD123, TCL1A and BDCA-2/CD303, as well as
TCF4), and without expressing myeloid, T-cell, or B-cell lineage markers. ${ }^{12}$ Based on the phenotype, BPDCN is further classified accordingly to maturation status as immature (CD34+), intermediate (CD117+ but CD34-) or mature (CD34- and CD117-). Intermediate and mature BPDCN are preferentially diagnosed from skin and nodal biopsies and immature BPDCN from bone marrow aspiration, but this classification does not provide any prognostic information. ${ }^{13}$ For minimal residual disease assessment purposes, an immunophenotypic characterization of reactive versus blastic PDC has been published which will undoubtedly help with diagnosis in bone marrow. ${ }^{14}$

Cytogenetic features include frequent loss of genetic material such as deletions (del5q31, del9p21.3, del13q13, del7p12, del12p), balanced chromosomal translocations, which are uncommon and not specific to the disorder with the notable exception of $\mathrm{t}(6 ; 8)$, involving SUPT3H and MYC loci. The landscape of adult BPDCN genomic alterations is dominated by TET2, ASXL1, NRAS, IKZF1 and NPM1 mutations.

Primary BPDCN is more common $(80-90 \%)$, but in $10-20 \%$ of the cases it develops in the context of hematologic malignancies, with molecular evidence of shared clonal origin in some case reports. Even rarer are cases that occur after chemotherapy for solid tumor or lymphoma. Moreover, myeloid neoplasms may present with associated mature $\mathrm{pDC}$ proliferation or a spectrum of $\mathrm{pDC}$ differentiation, but these entities are different from BPDCN. ${ }^{15}$

\section{Outcome and Response to Conventional Treatments}

Today, patients are still usually treated with ALL/lymphomatype or AML-type chemotherapy, and there is no approved standard treatment. With these treatments, overall survival barely exceeds 18 months, in spite of the achievement of an initial complete remission in more than half of the patients. ${ }^{16}$ Relapse is common, quick, and often lethal.

Prognostic factors of response to these therapies are mostly unknown, however a retrospective study has identified age $>60$ years, abnormal karyotype and terminal deoxynucleotidyltransferase (TdT) negativity in BPDCN cells at diagnosis as a factor of poorer outcomes. ${ }^{17}$

Autologous and allogeneic hematopoietic stem cell transplantations (HSCT) have been tried in this condition. They seem to improve overall survival in some cases, but only a few patients are eligible for this type of treatment due to the frequency of an advanced age and a rapidly 
progressing disease. Moreover, the real efficacy of HSCT in BPDCN cannot be precisely indicated because there are few reports and they include only small groups of patients. ${ }^{18}$ However, allogeneic stem cell transplantation seems to be a relevant therapeutic option if feasible in first complete remission. ${ }^{19}$

\section{Need for Novel Approaches: Emerging Targeted Therapies}

Therefore, there is a great need for novel therapies in BPDCN. In fact, drugs with a suitable toxicity profile for frail or elderly patients and which significantly improve survival in terms of the quality of life of patients are required.

BPDCN are CD4+CD56+ neoplasms defined by the lack of other cancer-specific cell surface markers, which emphasizes the difficulties finding an adequate target for a therapeutic strategy. CD38 is not always present at the cell surface (unlike reactive pDC). Therefore, CD123 is the most reliable marker to develop antibody-based therapies, such as monoclonal antibodies, antibody drug conjugates, dual affinity re-targeted agents or bispecific antibodies, or even chimeric antigen receptor T-cells.

Based on cytogenetic and molecular features of the disease, some potential therapeutic targets are emerging in BPDCN, among which are NF-kB pathway inhibitors (most often indirect inhibitors such as proteasome inhibitors, for example, bortezomib), DNA hypomethylating agents (5-azacytidine), BCL-2 inhibitors (venetoclax), bromodomain (BRD4) inhibitors, and folate metabolism inhibitors (pralatrexate). ${ }^{20}$ The development of precision medicinedriven combinations will be the only successful method to transform the prognosis of this disease. ${ }^{21,22}$ In this regard, recent evidence suggesting that azacytidine might increase BPDCN sensitivity to tagraxofusp through the restoration of the diphthamide synthesis pathway paves the way for conducting a clinical trial in myeloid malignancies. ${ }^{23}$

\section{Results of Tagraxofusp in the Treatment of BPDCN}

\section{Pharmacokinetics and Pharmacodynamics}

Tagraxofusp is administered intravenously, in a 15-minute infusion once daily for 5 days every three weeks. The area under the curve (AUC) for plasma concentration over time is $231 \mathrm{hr} \mathrm{mcg} / \mathrm{l}$ and Cmax is $162 \mathrm{mcg} / \mathrm{l}$. The mean volume of distribution is $5.1 \mathrm{~L}$. Mean terminal half-life is 0.7 hours. As a fusion protein with a molecular weight of 58
$\mathrm{kDa}$, tagraxofusp is expected to be eliminated via degradation and renal pathways. ${ }^{24}$

\section{Efficacy}

Viability assays in vitro and in vivo in a murine model have shown increased sensitivity of blastic plasmacytoid dendritic cells to tagraxofusp compared to other chemotherapeutic drugs. ${ }^{25}$

Currently, tagraxofusp is the only targeted therapy with a prospective evaluation of safety and efficacy in BPDCN, in two clinical trials. ${ }^{26,27}$ The main results concerning efficacy are shown in Table 1. Tagraxofusp, administered intravenously at a dose of 12 or $12.5 \mathrm{mg} / \mathrm{kg} /$ day for 5 days every 3 weeks, results in promising clinical activity in BPDCN, and in all the sites involved in the disease (skin, bone marrow, spleen, lymph node and viscera). More than half of the patients respond to the treatment. However, responses seem longer and more profound and the rate of complete response is higher in treatment-naive patients than in previously treated patients. Furthermore, it is an effective bridge to transplantation in front-line therapy. In the trial by Pemmaraju and colleagues, $45 \%$ of the patients treated with tagraxofusp in a first-line setting (12 $\mu \mathrm{g} / \mathrm{kg}$ ) were bridged to SCT during remission. Median overall survival (OS) was not reached in the group of treatment-naive patients, whereas median OS reached 8.5 months in previously treated patients, most of whom were not bridged to transplantation (one patient). Further studies are needed to assess the benefit of autologous or allogeneic stem cell transplantation after tagraxofusp.

It is of note that the benefit of tagraxofusp seems persistent in patients over 70 years old, with overall response and CR rates of $100 \%$ and $70 \%$ respectively in treatment-naive patients, and $70 \%$ and $10 \%$ respectively in previously treated patients.

\section{Toxicity Profile}

The toxicity profile of tagraxofusp in clinical trials is indicated in Table 1. Toxicities of grade 3 or higher are common and concerned 72 to $81 \%$ of the patients in prospective clinical trials.

Acute adverse events can occur during treatment or within a few hours after the infusion. They include chills, fever, nausea, and sometimes even hypoxemia and hypotension. These adverse events are mild to moderate in severity, transient, and easily manageable.

An increase in transaminases and thrombocytopenia are the most common grade $\geq 3$ toxicities. 


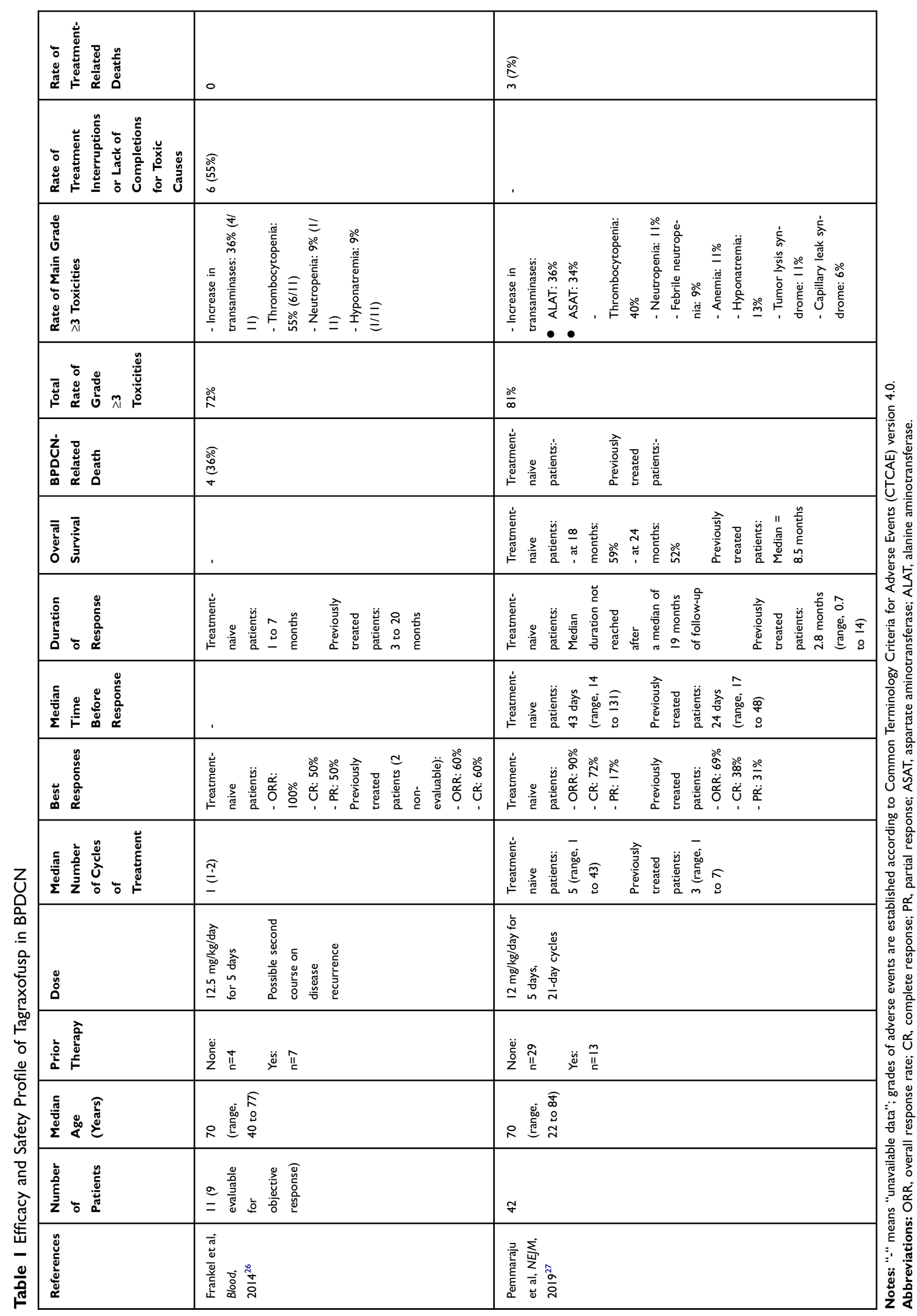


It must be emphasized that tumor lysis syndrome can occur.

Capillary leak syndrome (CLS) is a specific and frequent tagraxofusp toxicity. It occurs in $20 \%$ of patients, most frequently in the first week during the first treatment cycle. In most instances the severity is moderate and symptoms improve in a few days. However, severe or even lethal CLS has been reported after tagraxofusp. Since a decrease in serum albumin during the initial treatment days appeared to be the most consistent predictor of CLS, it should be strictly monitored. IV albumin supplementation is required to maintain serum albumin $\geq 3.2 \mathrm{~g} / \mathrm{dl}$ before tagraxofusp administration, and tagraxofusp should be withheld until CLS signs have resolved. Consequently, severe undernutrition and heart failure can promote CLS and interfere with treatment.

\section{Preliminary Results in Other Malignancies}

Tagraxofusp treatment is not specific to BPDCN. It has been tested in relapsed or refractory (R/R) CD123expressing myeloproliferative neoplasms, including AML and myeloproliferative neoplasms (MPN) such as chronic myelomonocytic leukemia (CMML) and myelofibrosis (MF). Related early-stage clinical trials are ongoing (NCT02268253, NCT02270463). Preliminary results have already been communicated.

In $18 \mathrm{R} / \mathrm{R}$ CMML patients, tagraxofusp showed 3 complete responses in bone marrow and $100 \%$ responses in spleen, including a $67 \%$ of reduction of $\geq 50 \%{ }^{28}$

In $27 \mathrm{R} / \mathrm{R}$ MF patients, tagraxofusp treatment significantly decreased symptoms in $45 \%$ of the patients, and reduced spleen size in $53 \%$ of the 17 evaluable patients with baseline splenomegaly. ${ }^{29}$

In both cases, the most frequent treatment-related adverse events were hypoalbuminemia ( 31 to $43 \%$ ), thrombocytopenia (24 to $30 \%$ ) and nausea (24 to $38 \%$ ). The most frequent grade $\geq 3$ adverse events were thrombocytopenia ( 8 to $13 \%$ ) and anemia (14 to $17 \%$ ). Capillary leak syndrome occurred in up to $19 \%$ of the patients, but the severity remained intermediate (grade 2).

Tagraxofusp has also been tested in other hematologic malignancies because of the high levels of CD123expressing plasmacytoid dendritic cells (pDCs) in the tumoral micro-environment but not at the tumoral cell surface. For instance, tagraxofusp has been used in a Phase $1 / 2$ trial in combination with pomalidomide and dexamethasone in relapsed or refractory multiple myeloma. ${ }^{30,31}$

Furthermore, tagraxofusp treatment tends not to be restricted to cancers since it has recently been used to target CD123-expressing pDCs in patients with systemic sclerosis. ${ }^{32}$ These cells are known to promote the inflammation and disease pathogenesis observed in patients with systemic sclerosis and lupus.

\section{Conclusion and Perspectives}

Tagraxofusp is effective in treating BPDCN, especially as a front-line therapy. It has shown promising clinical activity, with a high overall response rate and prolonged responses, even in elderly patients who are unsuitable candidates for stem cell transplantation. It seems to improve survival, but comparative trials are needed to affirm this. Grade $\geq 3$ adverse events are common. They are mainly represented by an increase in transaminases and thrombocytopenia. CLS can be severe and is an important adverse event to anticipate. As a result, tagraxofusp treatment requires preserved hepatic and cardiac functions, and strict monitoring of serum albumin.

Factors of a good response and prognostic factors after tagraxofusp treatment are unknown and should be defined. Similarly, the role of autologous and allogeneic stem cell transplantation after tagraxofusp should be specified.

No CNS involvement at diagnosis of BPDCN or at relapse was reported in patients treated with tagraxofusp. CNS involvement in BPDCN can occur in up $10 \%$ of patients. Therefore, the efficacy of tagraxofusp in treating or preventing this condition remains to be specifically assessed.

The efficacy of tagraxofusp in BPDCN may be increased in association with other cytotoxic drugs or targeted therapies. The high cost of the drug (26,660 US dollars $/ \mathrm{mL}$ ), and the absence of cost-effectiveness studies in real-world patients so far, highlights the need to develop a good biology-driven rationale to prescribe this drug in BPDCN and in other malignancies.

\section{Author Contributions}

All the authors contributed to data analysis, drafting or revising the article, gave final approval of the version to be published, and agree to be accountable for all aspects of the work.

\section{Disclosure}

The authors reports no conflicts of interest in this work. 


\section{References}

1. Muñoz L, Nomdedéu JF, López $\mathrm{O}$, et al. Interleukin-3 receptor $\alpha$ chain (CD123) is widely expressed in hematologic malignancies. Haematologica. 2001;86(9):1261.

2. Lucas N, Duchmann M, Rameau P, et al. Biology and prognostic impact of clonal plasmacytoid dendritic cells in chronic myelomonocytic leukemia. Leukemia. 2019;33(10):2466-2480. doi:10.1038/ s41375-019-0447-3

3. Jordan CT, Upchurch D, Szilvassy SJ, et al. The interleukin-3 receptor alpha chain is a unique marker for human acute myelogenous leukemia stem cells. Leukemia. 2000;14(10):1777-1784. doi:10.1038/sj.leu.2401903

4. Testa U, Riccioni R, Militi S, et al. Elevated expression of IL-3Ralpha in acute myelogenous leukemia is associated with enhanced blast proliferation, increased cellularity, and poor prognosis. Blood. 2002;100(8):2980-2988. doi:10.1182/blood-200203-0852

5. Testa U, Pelosi E, Frankel A. CD 123 is a membrane biomarker and a therapeutic target in hematologic malignancies. Biomark Res. 2014;2(1):1-11. doi:10.1186/2050-7771-2-4

6. Testa U, Pelosi E, Castelli G. CD123 as a therapeutic target in the treatment of hematological malignancies. Cancers. 2019;11(9):1358. doi:10.3390/cancers 11091358

7. Alkharabsheh O, Frankel AE. Clinical activity and tolerability of SL-401 (Tagraxofusp): recombinant diphtheria toxin and interleukin-3 in hematologic malignancies. Biomedicines. 2019;7 (1):6. doi:10.3390/biomedicines 7010006

8. Jen EY, Gao X, Li L, et al. FDA approval summary: tagraxofusp-erzs for treatment of blastic plasmacytoid dendritic cell neoplasm. Clin Cancer Res. 2020;26(3):532-536. doi:10.1158/1078-0432.CCR-19-2329

9. Garnache-Ottou F, Feuillard J, Ferrand C, et al. Extended diagnostic criteria for plasmacytoid dendritic cell leukaemia. Br J Haematol. 2009;145(5):624-636. doi:10.1111/j.1365-2141.2009.07679.x

10. Arber DA, Orazi A, Hasserjian R, et al. The 2016 revision to the World Health Organization classification of myeloid neoplasms and acute leukemia. Blood. 2016;127(20):2391-2405. doi:10.1182/blood2016-03-643544

11. Sapienza MR, Pileri A, Derenzini E, et al. Blastic plasmacytoid dendritic cell neoplasm: state of the art and prospects. Cancers. 2019;11(5):595. doi:10.3390/cancers11050595

12. Sukswai N, Aung PP, Yin CC, et al. Dual expression of TCF4 and CD123 is highly sensitive and specific for blastic plasmacytoid dendritic cell neoplasm. Am J Surg Pathol. 2019;43(10):1429-1437. doi:10.1097/PAS.0000000000001316

13. Martín-Martín L, López A, Vidriales B, et al. Classification and clinical behavior of blastic plasmacytoid dendritic cell neoplasms according to their maturation-associated immunophenotypic profile. Oncotarget. 2015;6(22):19204-19216. doi:10.18632/oncotarget.4146

14. Wang W, Khoury JD, Miranda RN, et al. Immunophenotypic characterization of reactive and neoplastic plasmacytoid dendritic cells permits establishment of a 10-color flow cytometric panel for initial workup and residual disease evaluation of blastic plasmacytoid dendritic cell neoplasm. Haematologica. 2020. doi:10.3324/haematol.20 20.247569

15. Hamadeh F, Awadallah A, Meyerson HJ, Beck RC. Flow cytometry identifies a spectrum of maturation in myeloid neoplasms having plasmacytoid dendritic cell differentiation. Cytometry B Clin Cytom. 2020;98(1):43-51. doi:10.1002/cyto.b.21761

16. Pagano L, Valentini CG, Grammatico S, Pulsoni A. Blastic plasmacytoid dendritic cell neoplasm: diagnostic criteria and therapeutical approaches. Br J Haematol. 2016;174(2):188-202. doi:10.1111/ bjh. 14146
17. Taylor J, Haddadin M, Upadhyay VA, et al. Multicenter analysis of outcomes in blastic plasmacytoid dendritic cell neoplasm offers a pretargeted therapy benchmark. Blood. 2019;134(8):678-687. doi:10.1182/blood.2019001144

18. Kharfan-Dabaja MA, Cherry M. Hematopoietic cell transplant for blastic plasmacytoid dendritic cell neoplasm. Hematol Oncol Clin North Am. 2020;34(3):621-629. doi:10.1016/j.hoc.2020.01.009

19. Kharfan-Dabaja MA, Reljic T, Murthy HS, Ayala E, Kumar A. Allogeneic hematopoietic cell transplantation is an effective treatment for blastic plasmacytoid dendritic cell neoplasm in first complete remission: systematic review and meta-analysis. Clin Lymphoma Myeloma Leuk. 2018;18(11):703-709.e1. doi:10.1016/j. clml.2018.07.295

20. Pemmaraju N. Novel pathways and potential therapeutic strategies for blastic plasmacytoid dendritic cell neoplasm (BPDCN): CD123 and beyond. Curr Hematol Malig Rep. 2017;12(6):510-512. doi:10.1007/s11899-017-0425-7

21. Zhang X, Sun J, Yang M, Wang L, Jin J. New perspectives in genetics and targeted therapy for blastic plasmacytoid dendritic cell neoplasm. Crit Rev Oncol Hematol. 2020;149:102928. doi:10.1016/j. critrevonc. 2020.102928

22. Deconinck E. Blastic plasmacytoid dendritic cell neoplasm: the european perspective. Hematol Oncol Clin North Am. 2020;34 (3):613-620. doi:10.1016/j.hoc.2020.01.012

23. Togami K, Pastika T, Stephansky J, et al. DNA methyltransferase inhibition overcomes diphthamide pathway deficiencies underlying CD123-targeted treatment resistance. J Clin Invest. 2019;129 (11):5005-5019. doi:10.1172/JCI128571

24. ELZONRIS US full prescribing information. Available from: https://elzonris.com/Content/documents/ELZONRIS_US_Full_ Prescribing_Information. Accessed May 29, 2020.

25. Angelot-Delettre F, Roggy A, Frankel AE, et al. In vivo and in vitro sensitivity of blastic plasmacytoid dendritic cell neoplasm to SL-401, an interleukin-3 receptor targeted biologic agent. Haematologica. 2015;100(2):223-230. doi:10.3324/haematol.2014.111740

26. Frankel AE, Woo JH, Ahn C, et al. Activity of SL-401, a targeted therapy directed to interleukin-3 receptor, in blastic plasmacytoid dendritic cell neoplasm patients. Blood. 2014;124(3):385-392. doi:10.1182/blood-2014-04-566737

27. Pemmaraju N, Lane AA, Sweet KL, et al. Tagraxofusp in blastic plasmacytoid dendritic-cell neoplasm. $N$ Engl J Med. 2019;380 (17):1628-1637. doi:10.1056/NEJMoa1815105

28. Patnaik MM, Ali H, Gupta V, et al. Results from ongoing phase $1 / 2$ clinical trial of tagraxofusp (SL-401) in patients with relapsed/refractory chronic myelomonocytic leukemia (CMML). Blood. 2018;132 (Supplement 1):1821. doi:10.1182/blood-2018-99-119302

29. Pemmaraju N, Gupta V, Ali H, et al. Results from a phase $1 / 2$ clinical trial of tagraxofusp (SL-401) in patients with intermediate, or high risk, relapsed/refractory myelofibrosis. Blood. 2019;134 (Supplement_1):558. doi:10.1182/blood-2019-131217

30. Ray A, Das DS, Song Y, et al. A novel agent SL-401 induces anti-myeloma activity by targeting plasmacytoid dendritic cells, osteoclastogenesis and cancer stem-like cells. Leukemia. 2017;31 (12):2652-2660. doi:10.1038/leu.2017.135

31. Richardson PG, Htut M, Gasparetto C, et al. Results from phase $1 / 2$ trial of tagraxofusp in combination with pomalidomide and dexamethasone in relapsed or refractory multiple myeloma. Blood. 2019;134(Supplement_1):3145. doi:10.1182/blood-2019-130410

32. Lindsay R, Chen J, Spiera R, Gordon J. CD123+ Plasmacytoid Dendritic Cells from Systemic Sclerosis Patients Are Susceptible to the Cytotoxic Activity of Tagraxofusp, a CD123-Targeted Therapy.:3. 2019:NJ USA: WILEY. 


\section{Publish your work in this journal}

OncoTargets and Therapy is an international, peer-reviewed, open access journal focusing on the pathological basis of all cancers, potential targets for therapy and treatment protocols employed to improve the management of cancer patients. The journal also focuses on the impact of management programs and new therapeutic agents and protocols on patient perspectives such as quality of life, adherence and satisfaction. The manuscript management system is completely online and includes a very quick and fair peer-review system, which is all easy to use. Visit http://www.dovepress.com/ testimonials.php to read real quotes from published authors.

Submit your manuscript here: https://www.dovepress.com/oncotargets-and-therapy-journal 